\title{
SPASER as a complex system: femtosecond dynamics traced by ab-initio simulations
}

\author{
Juan Sebastian Totero Gongora ${ }^{\mathrm{a}}$, Andrey E. Miroshnichenko ${ }^{\mathrm{b}}$, Yuri S. Kivshar ${ }^{\mathrm{b}}$, and Andrea \\ Fratalocchi ${ }^{\mathrm{a}}$ \\ aPRIMALIGHT, King Abdullah University of Science and Technology (KAUST), Thuwal \\ 23955-6900, Saudi Arabia \\ ${ }^{\text {b} N o n l i n e a r ~ P h y s i c s ~ C e n t r e, ~ A u s t r a l i a n ~ N a t i o n a l ~ U n i v e r s i t y, ~ C a n b e r r a ~ A C T ~ 2601, ~ A u s t r a l i a . ~}$
}

\begin{abstract}
Integrating coherent light sources at the nanoscale with spasers is one of the most promising applications of plasmonics. A spaser is a nano-plasmonic counterpart of a laser, with photons replaced by surface plasmon polaritons and the resonant cavity replaced by a nanoparticle supporting localized plasmonic modes. Despite the large body of experimental and theoretical studies, the understanding of the fundamental properties of the spaser emission is still challenging. In this work, we investigated the ultrafast dynamics of the emission from a core-shell spaser by developing a rigorous first-principle numerical model. Our results show that the spaser is a highly nonlinear system with many interacting degrees of freedom, whose emission sustain a rich manifold of different spatial phases. In the regime of strong interaction we observed that the spaser emission manifests an irreversible ergodic evolution, where energy is equally shared among all the available degrees of freedom. Under this condition, the spaser generates ultrafast vortex lasing modes that are spinning on the femtosecond scale, acquiring the character of a nanoparticle with an effective spin. Interestingly, the spin orientation is defined by spontaneous symmetry breaking induced by quantum noise, which is a fundamental component of our ab-initio model. This opens up interesting possibilities of achieving unidirectional emission from a perfectly spherical nanoparticle, stimulating a broad range of applications for nano-plasmonic lasers as unidirectional couplers, random information sources and novel form of photonics neural-networks.
\end{abstract}

Keywords: spaser, plasmonics, rotating dipole, nonlinear dynamics, unidirectional emission

\section{INTRODUCTION}

Many applications require the development of an integrable source of coherent light at the nanoscale. Among the possible candidates, the spaser is considered as one of the most promising solutions. ${ }^{1-3}$ In a spaser device a plasmonic resonance, namely a Surface Plasmon Polariton (SPP) of the structure, is amplified by the presence of a resonant medium. ${ }^{4}$ If the incident pumping power exceeds the spasing threshold, the spaser can sustain fully optical and coherent emission, which has been postulated theoretically and observed experimentally. ${ }^{5-7}$ From a theoretical perspective, great effort has been spent on providing a quantitative insight on the spasing threshold conditions. Neverthless, the theoretical understanding of the spaser is still challenging in many aspects. Classical and semi-classical methods, in fact, usually provide limited information on the temporal dynamics of the spontaneous/stimulated transition and on the evolution and mutual interaction of the spatial modes of the system before and during the lasing stage. The description of these dynamics requires the development of a rigorous time dependent method in which the amplyifing medium is treated in a fully quantum-mechanical framework. At the same time, a major experimental challenge in the understanding of the spaser dynamics lies in the difficulty of studying the detailed lasing emission of a single nanostructure. This information can provide essential data to get clear insights on the physics of the system. In order to address some of these challenges, in this work we investigate the dynamics underlying the emission from a single spaser by using massively parallel Finite-Differences Time-Domain (FDTD) ab-initio simulations. ${ }^{8}$ As a relevant case study, we considered a coreshell nanostructure in which a metallic core is coated with a shell of resonant material. The core-shell geometry

Further author information: (Send correspondence to J.S.T.G.)

J.S.T.G.: E-mail: js.totero@kaust.edu.sa, Telephone: +966546469564

Ultrafast Phenomena and Nanophotonics XX, edited by Markus Betz, Abdulhakem Y. Elezzabi, Proc. of SPIE Vol. 9746, 974618 · C 2016 SPIE · CCC code: 0277-786X/16/\$18 doi: $10.1117 / 12.2212967$

Proc. of SPIE Vol. 9746 974618-1 

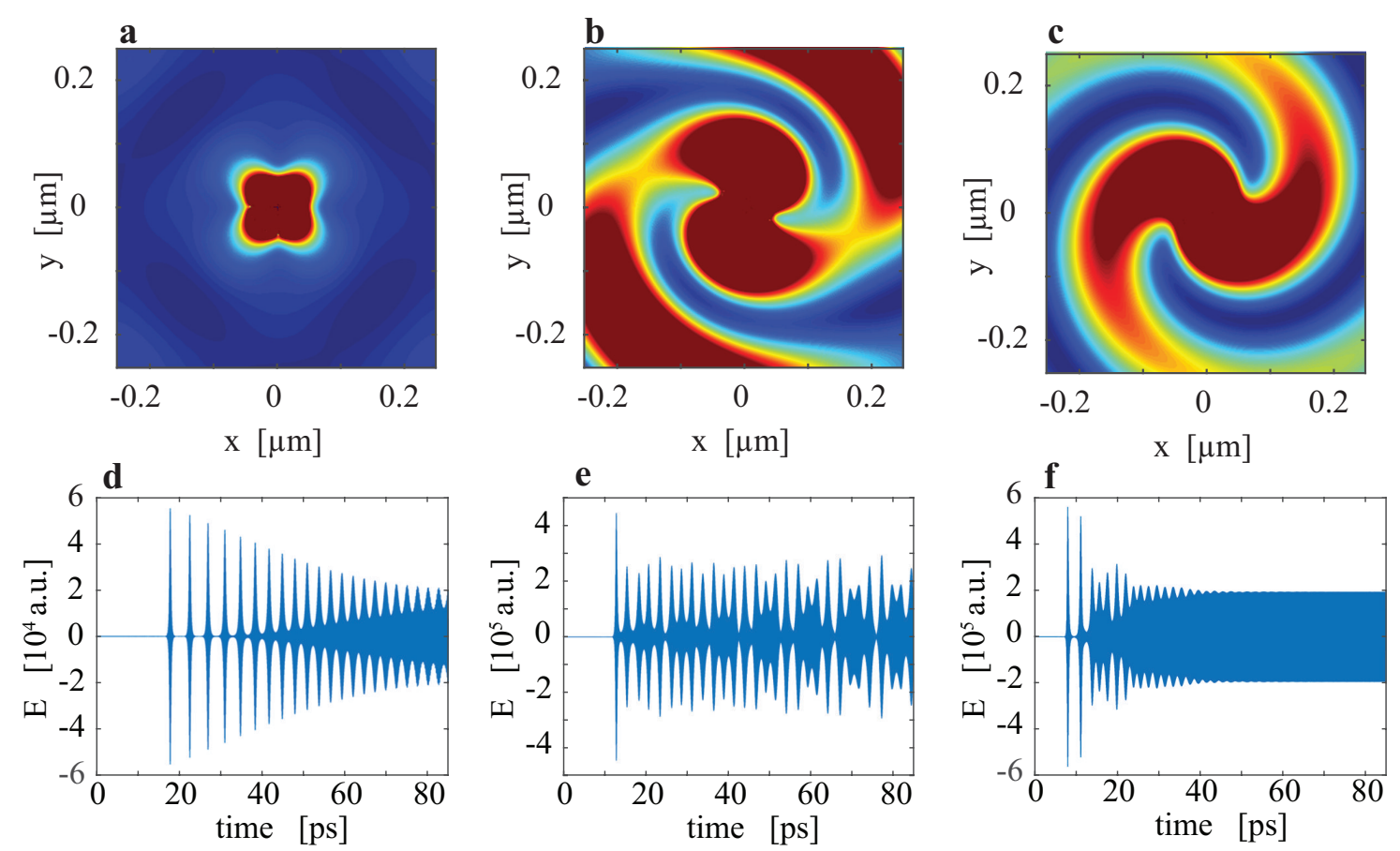

Figure 1. First-principle results on the spaser emission under different dye doping concentrations $N_{a}$. (a)-(c) Snapshot of the spatial distribution of electromagnetic energy. Panels (d)-(f) show the electric field evolution (normalized with respect to a reference value $E_{0}$ ) probed in proximity of the spaser. In the spinning phase (c,f), the energy emission of the spaser is $\approx 10$ times larger than in the multipole configuration (a,d).

allows for a precise design of the resonance spectrum just by varying the geometrical parameters of the structure and, therefore, is one of the most widely adopted configurations for the spaser. ${ }^{9,10}$ In order to simulate a spasing device, we implemented a full set of vectorial Maxwell-Bloch (MB) equations into the FDTD algorithm with a novel approach which explicitly considers the dispersion of the metal core. Interestingly, our simulations show that in the lasing regime the spaser emission is characterized by a novel type of rotational vortex-like patterns. The occurrence of a rotating emission opens new perspectives in the application of the spaser as a nanoscopic laser source, provided it can be exploited to produce unidirectional emission while preserving the spherical symmetry of the core-shell nanostructure.

\section{FDTD ANALYSIS OF THE SPASER EMISSION}

A rigorous ab-initio simulation of a core-shell spaser has to take into account the presence of both the metal core and the resonant medium. The response of the composite system is given by the total polarization field $\mathbf{P}_{T}$, which can be conveniently splitted into its linear and nonlinear contributions, namely $\mathbf{P}=\mathbf{P}_{\text {lin }}+\mathbf{P}_{N l i n}$. The linear polarization takes into account the linear dispersion of the media, i.e. $\mathbf{P}_{l i n}(t)=\varepsilon_{0} \int \mathrm{d} t^{\prime} \chi_{\text {lin }}\left(t-t^{\prime}\right) \mathbf{E}\left(t^{\prime}\right)$. The nonlinear polarization, conversely, describes the interaction between the electromagnetic field and the atoms composing the resonant medium. The evolution of the system can be studied by solving Maxwell's equations

$$
\left\{\begin{array}{l}
\frac{\partial \mathbf{H}}{\partial t}=-\frac{1}{\mu_{0}} \nabla \times \mathbf{E} \\
\frac{\partial \mathbf{E}}{\partial t}=\frac{1}{\varepsilon_{0}}\left[\nabla \times \mathbf{H}-\frac{\partial}{\partial t}\left(\mathbf{P}_{l i n}+\mathbf{P}_{n l i n}\right)\right]
\end{array}\right.
$$

with opportune additional equations for the polarization terms. ${ }^{8,11}$ In this work we model the resonant medium as a four-level atomic system with a ground state and three degenerate excited states, accounting for independent tra. The energy difference between the excited and ground states is set as $\hbar \omega_{0}$ and the quantum system is 
described using the density operator formalism. As a result, the evolution of the nonlinear polarization follows the Maxwell-Bloch (MB) equations, which in a three-dimensional space read

$$
\left\{\begin{array}{l}
\mathbf{P}_{N l i n}=e q_{0} N_{a}\left[S_{1} \hat{\mathbf{x}}+S_{4} \hat{\mathbf{y}}+S_{9} \hat{\mathbf{z}}\right] \\
\partial_{t} S_{l}=\sum \Gamma_{l m} S_{m}-\gamma_{l l}\left[S_{l}-S_{l}^{(0)}\right] \\
\Gamma_{l m}=\frac{j}{2 \hbar} \operatorname{Tr}\left\{H\left[\lambda_{l}, \lambda_{m}\right]\right\}, \gamma_{i j}=1 / \tau_{i j} .
\end{array}\right.
$$

Here $S_{j}$ is the j-th component of the Bloch coherence vector, $N_{a}$ is the density of polarizable atoms in the medium, $\lambda_{i}$ is the i-th generator of the $\mathrm{SU}(4)$ group, $\tau_{i j}$ are decay times and $H$ is the Hamiltonian of the atomic system. ${ }^{12}$ A full derivation of Eqs. (2) and the description of all the physical quantities can be found in. ${ }^{13,14}$ We solved Eqs. (1) and (2) inside the Finite-Differences-in-Time-Domain (FDTD) framework using our massively parallel FDTD simulator NANOCPP. ${ }^{15,16}$ More specifically, we used a novel computational approach which explicitly takes into account the presence of material dispersion. ${ }^{17}$ In our simulations the computational domain was organized as follows: the spaser was placed in the center of a box with $1 \mu \mathrm{m}$ side, and each spatial direction was discretized with 400 points (spatial resolution in air: $2.5 \mathrm{~nm}$ ). In order to simulate an open system, we used Uniaxial Perfectly Matched Layer (UPML) boundary conditions. ${ }^{8}$ Additionally, in our simulations we explicity considered the presence of quantum noise, whose amplitude is set in terms of the system temperature $T .{ }^{18}$ The geometry of the core-shell spaser is set as an Ag core embedded in a Rodhamine800-doped silica shell, which corresponds to a typical experimental configuration. ${ }^{6}$ In order to characterize the emission dynamics of the spaser, we conducted an extensive campaign of simulations for a two-dimensional core-shell structure and for different values of the pumping power $N_{a}$. Interestingly, above the spasing threshold, the emitted field is characterized by different spatial phases, which are shown in Figure 1. More specifically, for values of $N_{a}$ slightly above the spasing threshold, the emitted field is characterized by a single-mode stationary emission (Fig. 1a-d, $N_{a}=10^{27} \mathrm{part} / \mathrm{m}^{3}$ ). This regime corresponds to the standard spasing mechanism, with a single multi-polar mode being amplified by the resonant medium and achieving lasing. For increasing incident powers, however, the emitted field is characterized by the superposition of multipolar and rotational evolutions, corresponding to the competition and mutual interaction of different lasing modes (Fig. 1b-e, $N_{a}=2.25 \times 10^{27} \mathrm{part} / \mathrm{m}^{3}$ ). Finally, for values of $N_{a}>2.5 \mathrm{part} / \mathrm{m}^{3}$, the spaser emissions is fully stable and characterized by a purely rotational evolution with angular rotation equal to the emission frequency $\omega_{0}$ (Fig. 1-f), $N_{a}=3 \times 10^{27}$ ). Interestingly, due to to the presence of quantum noise, the direction of rotation of the emitted field changes randomly across different realizations of the spaser.

The occurrence of a spasing transition can be verified by measuring the spectral narrowing and the peak power as a function of the pumping rate, which are shown in Fig. 2. The spasing action is confirmed by the characteristic "S"-shaped behavior of the intensity peak versus density $N_{a}$ (green dots), as well as by the exponential narrowing of the emission linewidth (red dots), which approaches an almost constant value in the spasing regime. Interestingly, in the LL-plot the transition from a single-mode spasing to the fully rotational emission is marked by the occurrence of a seond high-power transition. The final linewidth of the spaser is of the order of $1-2 \mathrm{~nm}$, which is in quantitative agreement with experimental results. ${ }^{19,20}$ The similitude of Fig. 2 with Fig. 3b of, ${ }^{19}$ suggests the possibility that the spasing rotational dynamics can be observed within the pumping ranges of current experiments.

\section{CONCLUSIONS AND FURTHER DEVELOPMENTS}

We have investigated the ultrafast dynamics of the spaser by means of first-principle numerical methods. We have illustrated how the core-shell spaser, despite its simple geometry, shows a remarkable complexity that is able to sustain a rich scenario of different phases. While the theoretical analysis of the nonlinear dynamics underlying the rotational emission goes beyond the scope of this paper, it can be shown how our results open to the attractive perspective of using spasers as a new type of unidirectional sources. A fundamental problem in plasmonics is to achieve unidirectional light coupling, where the direction of propagation of surface plasmon polariton (SPP) waves can be controlled at the nanoscale. ${ }^{21}$ The possibility to obtain a stable rotating dipole emission in the spaser opens up the possibility to integrate unidirectional energy sources in spherically symmetric 
systems. In order to build a unidirectional launcher with the spaser, we took inspiration from the large literature on rotating dipole systems. ${ }^{22,23}$ Figure 3b-c illustrate two snapshot of the electromagnetic energy density for the structure of Fig. 3a, in which the spaser is placed near a metallic Ag plane. As seen in the figure, the light matter interaction between the spaser and the metal surface induces the generation of unidirectional surface plasmon polariton (SPP) waves, which propagate in the metal-dielectric interface by following the rotation direction of the energy emitted by the spaser. At the same time, another intriguing question is related to the mutual interaction of an ensemble of rotating spasers. Provided each rotating spaser can be interpreted as a nanoparticle with an "effective" spin, an ensemble of spasers represents an ideal candidate for a random multi-spin system. Systems with random spins, which is considered as the archetype of a complex system, have inspired a large number of fascinating applications, ranging from novel classes of error correcting codes, ${ }^{24}$ to new concepts in neurobiology and brain functions. ${ }^{25}$

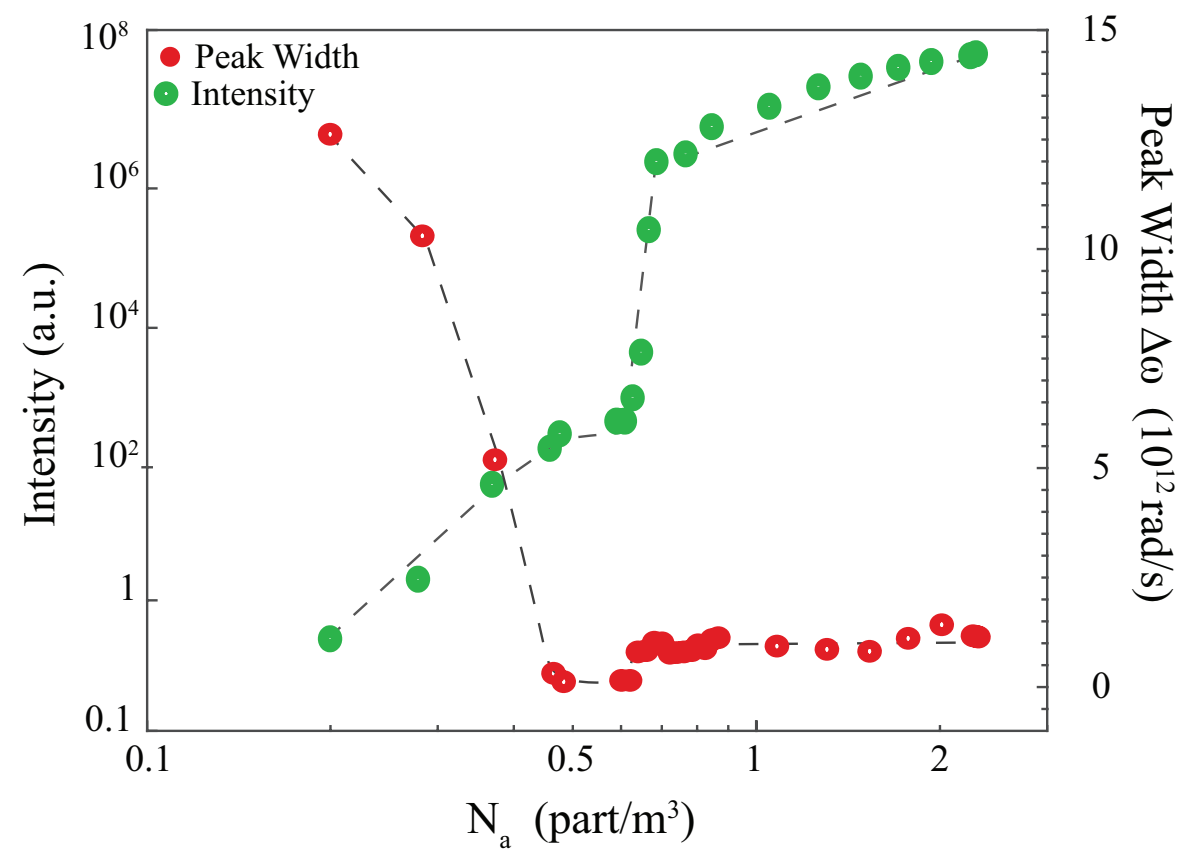

Figure 2. Lasing characterization and spasing threshold. Double logarithmic LL plot of lasing intensity (green circles) and emission linewidth (red circles). In the multipole lasing emission region, we observe the simultaneous occurrence of a nonlinear enhancement of the lasing intensity and the formation of a plateau in the spectral narrowing, which are the characteristic signature of a spasing transition. These results are in accordance with the experimentally observed transitions for single spaser structures. ${ }^{19,20}$

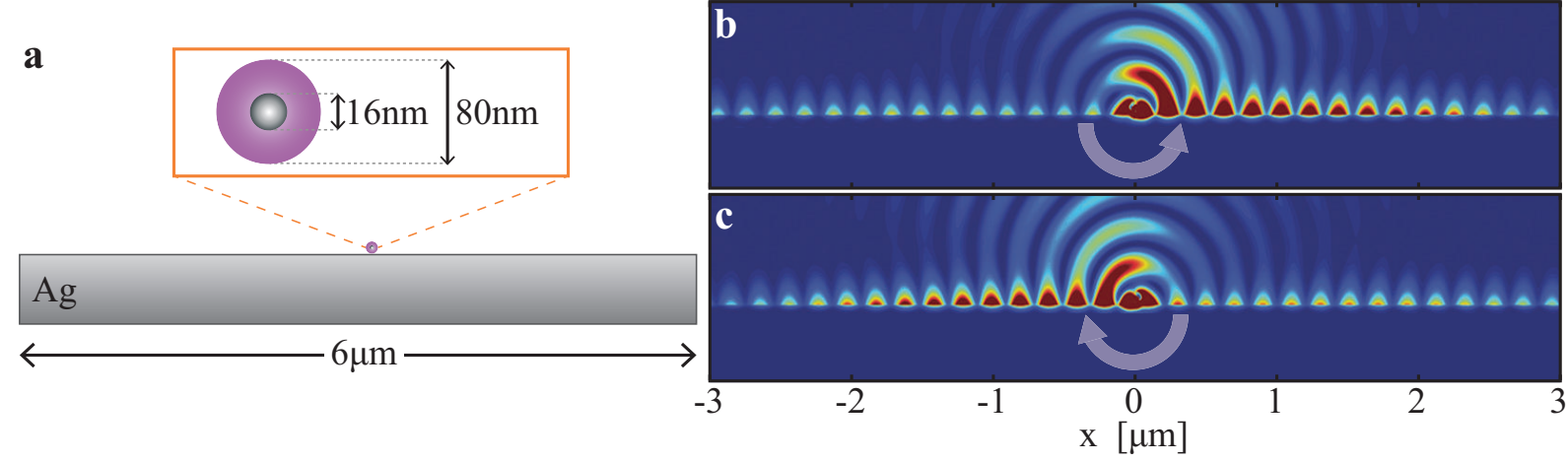

Figure 3. The rotating spaser as unidirectional energy launcher. In panel (a), we considered a spaser placed in proximity of a metallic Ag surface (a, left). According to the spin orientation acquired by the laser in the rotational phase, energy is coupled on SPP wave trains that propagate in different directions along the $x$ axis (panels b and c). 


\section{Acknowledgment}

For the computer time, we have used the resources of the KAUST Supercomputing Laboratory. This work is part of the research program of Kaust "Optics and plasmonics for efficient energy harvesting" (Award No. CRG-1-2012-FRA-005) and it was also supported by the Australian Research Council.

\section{REFERENCES}

[1] Rupasinghe, C., Rukhlenko, I. D., and Premaratne, M., "Spaser made of graphene and carbon nanotubes," ACS Nano 8(3), 2431-2438 (2014).

[2] Ma, R.-M., Ota, S., Li, Y., Yang, S., and Zhang, X., "Explosives detection in a lasing plasmon nanocavity," Nat. Nanotechnol. 9, 600-604 (08 2014).

[3] Sidiropoulos, T. P. H., Roder, R., Geburt, S., Hess, O., Maier, S. A., Ronning, C., and Oulton, R. F., "Ultrafast plasmonic nanowire lasers near the surface plasmon frequency," Nat. Phys. 10, 870-876 (09 2014).

[4] Bergman, D. J. and Stockman, M. I., "Surface plasmon amplification by stimulated emission of radiation: Quantum generation of coherent surface plasmons in nanosystems," Phys. Rev. Lett. 90, 027402-027405 (Jan 2003).

[5] Zheludev, N. I., Prosvirnin, S. L., Papasimakis, N., and Fedotov, V. A., "Lasing spaser," Nat. Photon. 2, 351-354 (06 2008).

[6] Noginov, M. A., Zhu, G., Belgrave, A. M., Bakker, R., Shalaev, V. M., Narimanov, E. E., Stout, S., Herz, E., Suteewong, T., and Wiesner, U., "Demonstration of a spaser-based nanolaser," Nature 460, 1110-1112 (08 2009).

[7] Zhang, Q., Li, G., Liu, X., Qian, F., Li, Y., Sum, T. C., Lieber, C. M., and Xiong, Q., "A room temperature low-threshold ultraviolet plasmonic nanolaser," Nat. Commun. 5 (09 2014).

[8] Taflove, A. and Hagness, S. C., [Computational electrodynamics : the finite-difference time-domain method], Artech House antennas and propagation library, Artech House, Boston, 3rd ed. (2005).

[9] Liu, W., Miroshnichenko, A. E., Neshev, D. N., and Kivshar, Y. S., "Broadband unidirectional scattering by magneto-electric core-shell nanoparticles," ACS Nano 6(6), 5489-5497 (2012).

[10] Kristensen, P. T. and Hughes, S., "Modes and mode volumes of leaky optical cavities and plasmonic nanoresonators," ACS Photonics 1(1), 2-10 (2014).

[11] Taflove, A., Oskooi, A., and Johnson, S. G., [Advances in FDTD computational electrodynamics : photonics and nanotechnology], Artech House antennas and propagation series, Artech House (2013).

[12] Ziolkowski, R. W., Arnold, J. M., and Gogny, D. M., "Ultrafast pulse interactions with two-level atoms," Phys. Rev. A 52(4), 3082-3094 (1995).

[13] Fratalocchi, A., Conti, C., and Ruocco, G., "Three-dimensional ab initio investigation of light-matter interaction in mie lasers," Physical Review A 78(1) (2008).

[14] Conti, C. and Fratalocchi, A., "Dynamic light diffusion, three-dimensional anderson localization and lasing in inverted opals," Nat. Phys. 4, 794-798 (10 2008).

[15] Coluccio, M. L., Gentile, F., Das, G., Nicastri, A., Perri, A. M., Candeloro, P., Perozziello, G., Proietti Zaccaria, R., Gongora, J. S. T., Alrasheed, S., Fratalocchi, A., Limongi, T., Cuda, G., and Di Fabrizio, E., "Detection of single amino acid mutation in human breast cancer by disordered plasmonic self-similar chain," Science Advances 1(8) (2015).

[16] Liu, C., van der Wel, R., Rotenberg, N., Kuipers, L., Krauss, T., Di Falco, A., and Fratalocchi, A., "Triggering extreme events at the nanoscale in photonic seas," Nature Physics (2015).

[17] Gongora, J. S. T. and Fratalocchi, A., [Computational Chemistry Methodology in Structural Biology and Material Sciences], ch. Ab-Initio Techniques For Light Matter Interaction At The Nanoscale, Apple Academic Press, Oakville (2015).

[18] Andreasen, J., Cao, H., Taflove, A., Kumar, P., and Cao, C.-q., "Finite-difference time-domain simulation of thermal noise in open cavities," Phys. Rev. A 77, 023810 (Feb 2008). 
[19] Lu, Y.-J., Kim, J., Chen, H.-Y., Wu, C., Dabidian, N., Sanders, C. E., Wang, C.-Y., Lu, M.-Y., Li, B.-H., Qiu, X., Chang, W.-H., Chen, L.-J., Shvets, G., Shih, C.-K., and Gwo, S., "Plasmonic Nanolaser Using Epitaxially Grown Silver Film," Science 337, 450-453 (July 2012).

[20] Ho, J., Tatebayashi, J., Sergent, S., Fong, C. F., Iwamoto, S., and Arakawa, Y., "Low-threshold near-infrared gaasalgaas coreshell nanowire plasmon laser," ACS Photonics 2(1), 165-171 (2015).

[21] Miroshnichenko, A. E. and Kivshar, Y. S., "Polarization traffic control for surface plasmons," Science 340(6130), 283-284 (2013).

[22] Mueller, J. P. B. and Capasso, F., "Asymmetric surface plasmon polariton emission by a dipole emitter near a metal surface," Phys. Rev. B 88, 121410 (Sep 2013).

[23] Rodríguez-Fortuño, F. J., Marino, G., Ginzburg, P., O'Connor, D., Martínez, A., Wurtz, G. A., and Zayats, A. V., "Near-field interference for the unidirectional excitation of electromagnetic guided modes," Science 340(6130), 328-330 (2013).

[24] Sourlas, N., "Spin-glass models as error-correcting codes," Nature 339, 693-695 (1989).

[25] Langston, R. F., Ainge, J. A., Couey, J. J., Canto, C. B., Bjerknes, T. L., Witter, M. P., Moser, E. I., and Moser, M.-B., "Development of the spatial representation system in the rat," Science 328(5985), 1576-1580 (2010). 